\title{
ARTRÓPODES ASSOCIADOS A EXCREMENTOS EM AVIÁRIOS
}

\section{THORA LILLY AAGESEN}

Orientador: Prof. Dr. EVONEO BERTI FILHO

Dissertação apresentada à Escola Superior de Agricultura "Luiz de Queiroz", da Universidade de São Paulo, para obtenção do título de Mestre em Ciências Biológicas. Area de Concentração: Entomologia.

P I R A C I C A B A

Estado de São Paulo - Brasil

Agosto - 1988 
Aagesen, Thora Lilly

A11la Artrópodes associados a excrementos em aviários. Piracicaba, 1988.

$38 p$.

Diss. (Mestre) - ESALQ

Bibliografia.

1. Artrópode para controle biológico 2. Áviário Praga - Controle biológico-3. Mosca - Controle bioló gico I. Escola Superior de Agricultura Luiz de Queiroz, Piracicaba 
ii.

ARTRÓPODES ASSOCIADOS A EXCREMENTOS EM AVIARIOS

THORA A ILILY AAGESEN

Aprovada em 08/09/1988

Comissão julgadora:

Prof. Dr. Evoneo Berti Filho

ESALQ/USP

Prof. Dr. José Djair Vendramim ESALQ/USP

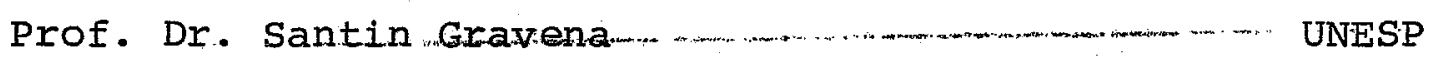

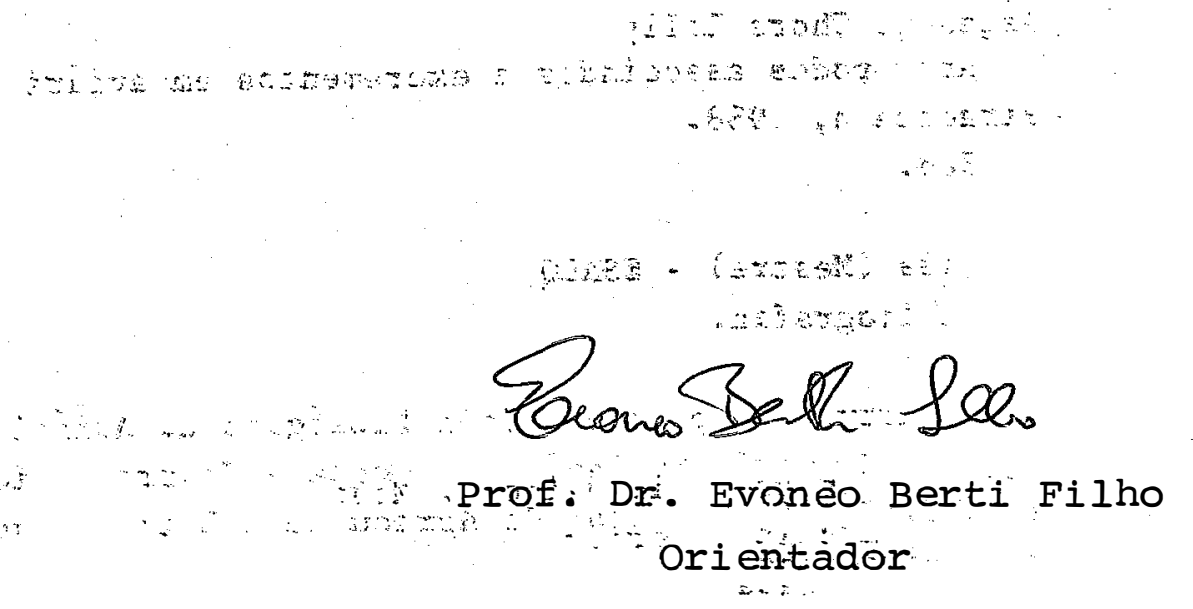


iii.

Aos meus pais

e

ao meu esposo,

DEDTCO,

Aos meus colegas de trabalho, OFEREÇO 


\section{AGRADECIMENTOS}

- Ao Prof. Dr. Evoneo Berti Filho, pela orientação e incentivo recebidos no decorrer desta pesquisa; pela versão do resumo para o inglês, e pela amizade e con sideração;

- À FAPESP, pela bolsa concedida;

- $\bar{A}$ Granja Mizumoto, na pessoa de Joaquim Gobara, pelo estímulo, interesse e valiosa colaboração, viabilizan do a execução desta pesquisa;

- Ao Dr. Howard Frank, da "University of Florida", Gainesville, pela identificação dos estafilinídeos;

- Ao Dr. Rupert L. Wenzel, do "Field Museum of Natural History", Chicago, pela identificação dos histerídeos;

Ao Dr. Fred D. Bennett, pelo auxílio prestado na identificação do material;

- Aos Professores do Departamento de Entomologia da ESALQ/USP, pelos ensinamentos e amizade.

- Aos funcionários do Departamento de Entomologia da ESALQ/USP e do Laboratório de Controle Biológico de Mosca domēstica, particularmente ao Lilson Cēsar da Silva, pela colaboração e interesse;

- Aos colegas do Curso, pela amizade e incentivo, partí cularmente à Antonia Barcelos Correia, Valmir Antonio Costa, Celso Omoto, Francisco Lozano Leonel Júnior, Willy Chiaravalle, Miguel Neira e Carlos Eduardo Masís Chacōn, pela compreensão e respeito e à Teresinha Augusta Giustolin pela colaboração. 


\section{INDICE}

Pāgina

LISTA DE TABELAS ....................... vi

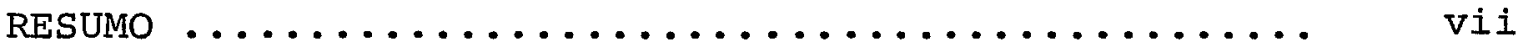

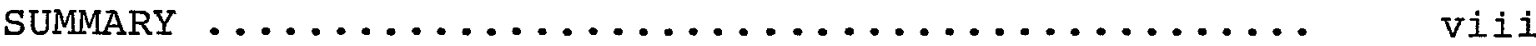

1. INTRODUÇÃO $\ldots \ldots \ldots \ldots \ldots \ldots \ldots \ldots \ldots \ldots \ldots \ldots \ldots \ldots \ldots \ldots$

2. REVISÃo DE IITERATURA ..................... 4

2.1. Principais predadores ................. 4

2.1.1. Coleoptera ................... 4

2.1.2. Hymenoptera ................. 7

2.1.3. Diptera ..................... 8

2.1.4. Outros artrópodes .............. 9

2.2. Mētodos de coleta ................... 13

3. MATERIAL E METODOS ...................... 14

3.1. Descrição da Granja .................. 14

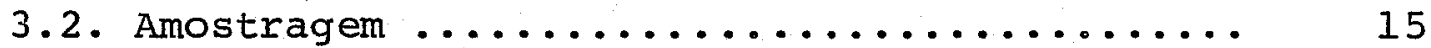

3.3. Extração da fauna do excremento amostrado .. 15

3.4. Triagem e identificação ............... 16

4. RESULTADOS E DISCUSSÃO ................... 18

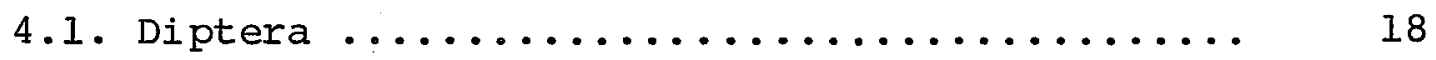

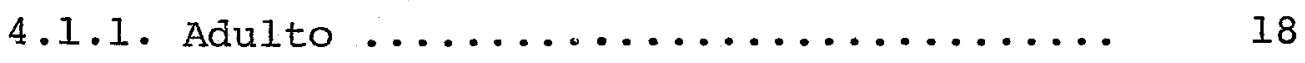

4.1 .2 . Imaturo ..................... 22

4.2. Coleoptera ........................ 25

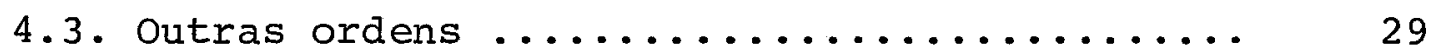

4.4. Outros artrópodes .................. 30

5. CONCLUSÕES $\ldots \ldots \ldots \ldots \ldots \ldots \ldots \ldots \ldots \ldots \ldots \ldots \ldots \ldots \ldots$

REFERENCIAS BIBLIOGRĂFICAS ............... 32 
vi.

LISTA DE TABELAS

Pāgina

Tabela no

1. Familias de dípteros adultos coletados em excrementos de aves. Número de indivíduos coletados e porcentagem. Bastos-SP, setembro/1985 a dezembro/1986 ......................

2. Espēcies mais abundantes de dípteros adultos, coletados em escremento de aves. Bastos-SP, se tembro/1985 a dezembro/1986...............

3. Famílias e espécies de dípteros, estāgio larval, coletados em excremento de aves. Número

de indivíduos coletados e porcentagem. Bastos-SP, setembro/ 1985 a dezembro/1986........

4. Porcentagem mensal de larvas de M. domestica e H. ellucens, coletadas em excremento de aves. Bastos-SP, setembro/1985 a dezembro/1986.....

5. Familias mais abundantes de coleópteros adultos coletados em excremento de aves. Nümero de individuos coletados e porcentagem. BastosSP, setembro/1985 a dezembro/1986...........

6. Espēcies mais abundantes de coleópteros coleta dôs em excremento de aves. Nümero de indivíduos e porcentagem. Bastos-SP, setembro/1985 a dezembro/ $1986 \ldots \ldots \ldots \ldots \ldots \ldots \ldots \ldots \ldots \ldots \ldots \ldots \ldots$ 


\title{
ARTRÓPODES ASSOCIADOS A EXCREMENTOS EM AVIÁRIOS
}

\author{
Autora: THORA LILLY AAGESEN
}

Orientador: Prof. Dr. EVONEO BERTI FILHO

\section{RESUMO}

Esta pesquisa teve por objetivo efetuar um levantamento dos artrópodes predadores de Musca domestica (Muscidae) que ocorrem em excremento de aves, na região de BastosSP, para incrementar programas de manejo integrado de moscas en aviārios. Amostras semanais foram tomadas do excremento acumulado sob as gaiolas, no decorrer de dezesseis meses, no período de setembro de 1985 a dezembro de 1986. O mëtodo empreagado constituiu de coletas de amostras do meio, com um cí lindro (10 cm de diâmetrox $12 \mathrm{~cm}$ de altura) e posterior extração da fauna, com auxílio do funil de "Tullgren". Os principais predadores coletados foram da familia Histeridae representada por seis espécies e Staphylinidae, por doze espécies. Outros predadores foram encontrados: Hyärophilidae (Coleoptera), Muscidae, Scenopidae (Diptera), Formicidae (Hymenoptera), Anthocoridae (Hemiptera), ācaros das famílias Macrochelidae e Uropodidae e Pseudoscorpionida. 


\section{ARTHROPODS ASSOCIATED WITH POULTRY MANURE}

Author: THORA LILLY AAGESEN Adviser: Prof. Dr. EVONEO BERTI FILHO

\section{SUMPARY}

This research deals with a survey of arthropod predators of Musca domestica I. in poultry droppings, aiming at a program of integrated house fly management in poultry areas. The study was carried out in the Granja Mizumoto, in Bastos, State of São Paulo, Brazil. A cylinder (10 cm diameter $\mathrm{x} 12 \mathrm{~cm}$ height) was used to take samples from the poultry droppings, each week, from september 1985 to December 1986 . The material of the samples was placed in Tullgren funnels to extract the fauna. The following predators were recorded: Histeridae, Staphylinidae and Hydrophilidae (Coleoptera), Muscidae, Scenopidae (Diptera), Formicidae (Hymenoptera), Anthocoridae (Hemiptera), Macrochelidae and Uropodidae (Acarina) and Pseudoscorpionida. 


\section{INTRODUCÃO}

Musca domestica L., 1758 è uma espēcie cosmopo lita, não ocorrendo apenas nas regiões de grande altitude e nas polares.

Sua dispersão e distribuição pelo mundo foi al tamente favorecida por ser sinantrópica e onívora. Deste modo, torna-se de grande importância médico-veterinária, pois entra freqüentemente em contato com agentes patogênicos de lixos e excrementos, tornando-se ótimo vetor de vírus, rickettsias, protozoārios e bactērias.

Nas regiões onde granjas avícolas situam-se prō ximas às áreas urbanizadas, os vários dípteros que se criam no esterco, produzido pelas aves, invadem as cidades, representando um sērio problema sanitārio.

Além de constituírem uma ameaça à saúde püblica e animal, as moscas causam problemas econômicos para o avi cultor, comprometendo a produção e a qualidade dos ovos.

O uso de produtos químicos, como método de con trole tem se mostrado ineficaz, pois aliado à alta capacidade reprodutiva das moscas, o uso constante de inseticidas, tem favorecido a seleção de indivíduos resistentes além 
de possibilitar o aparecimento de residuos químicos nos ovos e na carne das aves.

Torna-se evidente, portanto, a necessidade do desenvolvimento de mētodos alternativos.

Sob o ponto de vista da Entomologia moderna, o sucesso do controle de uma praga está intimamente relacionado com a associação dos vários métodos existentes.

Técnicas de manejo do excremento, juntamente com técnicas de controle químico, prātica sanitāria, controle biológico e outras, constituem os instrumentos para o manejo integrado de Musca domestica em aviārios.

E no controle biológico que a prática do manejo integrado tem um dos seus principais suportes.

Parasitóides e predadores entomófagos exercem, no seu habitat, um papel importante na regulação da abun dância dos insetos pragas. Esta complexa associação pode envolver muitas espécies, cada qual com uma importância variāvel, dependente de tempo e lugar.

A prática do controle biológico sobre uma popu lação requer estudos bāsicos que envolvam aspectos biológicos, ecológicos, taxonômicos, fisiológicos, genéticos e demográficos, comportamentais, nutricionais e métodos de criação, tanto do inseto praga visado, como de seus inimigos naturais.

Este träbalho tem por objetivo efetuar um le- 
vantamento dos artrópodes predadores de Musca domestica que ocorrem em excremento de aves, na região de Bastos-SP, com o intuito de fornecer subsídios para o conhecimento da sua feno logia, que possam ser utilizados na elaboraçãode programas de manejo integrado em aviários. 


\section{REVISÃO DE LITERATURA}

Musca domestica L. sofre ataques de numerosos agentes predadores, que causam uma significativa redução em sua população.

\subsection{PRINCIPAIS PREDADORES}

\subsubsection{Coleoptera}

Todos os estafilinídeos Philonthus spp. são predadores, especialmente de larvas de dipteros, encontrados em matēria orgānica em decomposição (XAMBEAU, 1907).

MANK (1923) criou larvas de Philonthus spp. com ācaros e larvas de dípteros e EVANS (1964) descreveu o método de criação de adulto de philonthus decorus.

Staphylinus maxillosus villosus Grav. (Staphylinidae) è um coleópteto comum e conspícuo, considerado necrôfago, e consome grande número de larvas (DAVIS, 1915 e FITCHER, 1949). FITCHER (1949) relatou que adultos famintos poderiam consumir acima de vinte larvas sucessivamente; 
não è mencionado, entretanto, o tamanho da larva ou a espécie. Adultos também consomer formigas, térmitas, minhocas, alēm de suas próprias larvas e outras larvas.

ABвоTT (1937) estudou o desenvolvimento e a biologia de $S$. maxillosus villosus. o autor observouque a es pécie é de natureza altamente predatória de ambos, adulto e larva, como tambēm de comportamento agressivo uns com os outros.

SANDERS \& DOBSON (1966) observaram larvas e adultos do estafilinideo Philonthus cruentatus alimentando-se de larvas de dipteros, sobre excremento bovino. Além desta es pécie, os autores encontraram outros coleópteros predadores das familias Histeridae, Hydrophilidae e staphylinidae.

Nos estudos de comportamento alimentar e poten cial de predação de algumas espécies predadoras, PECK observou que adultos de carcinops pumilio (Histeridae) consomem principalmente ovos de $M$. domestica enquanto adultos de Philonthus politus e $S$. maxillosus villosus, predam todos os estăgios imaturos.

Segundo BALDUF (1935), histerídeos são comumen te encontrados em plantas em decomposição, excremerito e car ne putrefata.

Muitas espécies de histerídeos vivem sob casca de árvores, em ninhos de formigas e tērmitas, em ninhos ou re fúgio de aves ou mamíferos, e outros estão associados com fun 
gos, carcaça ou excremento animal (DILLON \& DILLON, 1972). De acordo com АВВОTТ (1937), a maioria è necrófaga.

Wenzel $(1968)^{1}$, citado por PECK (1968) afirmou que todas as larvas de histerídeos são predadoras.

SIMMONDS(1940) relatou que certos coleópteros das familias Copridae e Histeridae foram eficientes na redução de muscóides em excremento bovino em Java, Sumatra e Afri ca do Sul.

No levantamento e estudo realizadospor PECK \& ANDERSON (1969), da fauna de predadores em excremento de aves, foi observado que os coleópteros predadores afetam principalmente a população de. M. domestica e, em menor proporção, a população de Fannia canicularis (Muscidae).

Estudos realizados por LEGNER \& OLTON (1970) so bre a distribuição de insetos predadores e escavadores em excremento animal, no sul da Califórnia, mostraram diferença nu mērica de espēcies coletadas em três tipos de excremento. A maior variedade foi encontrada em esterco de galinha, seguido do bovino e eqüino. Além do tipo de excremento, o clima pode influenciar, tornando-se um fator decisivo na limitação da distribuição de algumas espēcies.

PECK \& ANDERSON (1970) determinaram quão rāpi-

I WENZEL, R. L. (Comunicação pessoal), 1968. 
da uma nova camada de esterco reconstitui sua fauna, bem como a influência exercida sobre as larvas de vārios dípteros e seus predadores, na remoção periódica do mesmo. Os autores observaram que o aumento populacional de ácaros predadores, apōs a remoção, deu-se de maneira lenta e o aparecimento dos coleópteros predadores foi bastante retardado.

LEGNER et alii (1975) avaliaram a densidade, distribuição e interação dos predadores e escavadores encontrados em excremento de aves. Os autores sugeriram, apōs analisarem as correlações entre predadores e presa, que as ativi dades dos predadores influenciaram periodicamente os fatores estudados.

GEETHA BAI \& SANKARAN (1977) consideram os his terídeos e o estafilinídeo Aleochara puberula bastante promis sores no controle biológico de moscas.

LEGNER et alii (1981) observaram que em estrume bovino a grande biomassa de predadores e escavadores habitam excremento de até oito dias de idade nos quais encontram-se, tambēm, ovos e larvas de dípteros.

\subsubsection{Hymenoptera}

Phillips $(1934)^{2}$, citado por LEGNER \& BRYDON

2 PHILIIPS, J.R. The biology and distribution of ants in Hawaiian pineapple fields. Hawaii Pineapple Prod. Sta. Bull. , 15: 1-57, 1934. 
(1966) referiu-se a Pheidole megacephala (Formicidae) como um inseto eficiente no controle de M. domestica nas Ilhas Havaianas, mesmo apresentando o inconveniente de destruir certos insetos benéficos. SIMMONDS (1940) tambēm se referiu à mesma espécie que, quando introduzida acidentalmente em Fiji, em 1910, promoveu um controle efetivo da mosca, por vários anos.

Outras formigas contribuem no controle natural de M. domestica, alimentando-se dos ovos. PIMENTEL (1955), em Porto Rico, atribuiu 91\% do controle de M. domestica à Solenopsis gemminata (Formicidae).

\subsubsection{Di ptera}

Portchinsky $(1913)^{3}$, citado por PECK \& ANDERSON (1969), mostrou que larvas de outros dípteros (Muscina sp.) (Muscidae) são efetivas na destruição de larvas de Musca.

PECK \& ANDERSON (1969) relataram a atividade predatória de Muscina stabulans sobre larvas de Fannia canicu laris e observaram, tambëm, sua maior ocorrência na primavera.

A primeira constatação que larvas de Ophyra leu costoma Wiedmann (Muscidae) são predadoras foi feita por séguy (1923) ${ }^{4}$,

3 PORTCHINSKY, T. Muscina stabulans Fall mouche nuisible à 1 'horme et à son ménage, en état larvaire destructeuse des larves de Musca domestica.

4 Thavaux du Bureau Entomol. du Dept. d'Agric., 10(1): 1-39, 1913. SEGUY, E. Diptères anthomyides. Faune de France, no 6, 1923. 393p. 
citado for PECK (1968), que observou larvas predando outros insetos que viviam no mesmo habitat.

ANDERSON \& POORBAUGH (1964a) e PECK \& ANDERSON (1969) discutiram o aspecto benéfico de 0. leucostoma, predador de Fannia e Musca no estágio larval.

ANDERSON \& POORBAUGH (1964b) reportaramque lar vas de 0 . leucostoma são altamente vorazes, matando mais de 20 pequenas larvas de mosca doméstica, por dia. Os autores ob servaram que larvas de 0 . leucostoma usari suas peças bucais agudas para injetar uma toxina paralisante na vítima. Eles averigüaram que 0 . leucostoma destrói larvas de M. stabulans, M. domestica, F. canicularis e Aldrichina grahami (Calliphoridae). PECK (1969) avaliou a capacidade predatória de o. Leucostoma e constatou seu elevado potencial.

\subsubsection{Outros artrópodes}

Legner $(1965)^{5}$, citado por LEGNER et alii (1975), observou dermápteros em esterco animal alinentando-se vorazrente de ovos, larvas e pupas de M. domestica.

LEGNER \& OLTON (1970) e LEGNER et alii (1975;

5 LEGNER, E.F. Un complejo de los artropodos que influyen en los estadios juvenilles de Musca domestica I. en Puerto Rico. Carib. J. Sci., 5: $109-15,1965$. 
1981) relataram a presença de dermápteros e hemípteros predadores de larvas de Musca sp. associados a esterco animal, em vãrias regiões do mundo.

PEREIRA \& DE CASTRO (1945) foram os primeiros a observar que Macrocheles muscaedomesticae Scopoli (Acarina, Macrochelidae) preda M. domestica.

Filipponi (1955) ${ }^{6}$, citado por PECK (1968), tes tando a capacidade predatória de M. muscaedomesticae ofereceu ovos e larvas do primeiro $\left(I_{1}\right)$, segundo $\left(I_{2}\right)$ e terceiro $\left(I_{3}\right)$ Instar de mosca doméstica em um cilindro com papel de filtro umedecido, às fêmeas de ácaros, não alimentadas por 2 a 8 dias. Uma média entre 2,8 e 4,6 ovos foram consumidos nas primeiras duas horas. Neste periodo predou $2,6 \mathrm{~L}_{1}$ e em 5 horas $0,78 \mathrm{~L}_{2}$. Os àcaros não foram capazes de predar larvas do terceiro ins$\operatorname{tar}\left(L_{3}\right)$ e pupas.

RODRIGJEZ \& WADE (1961) observaram que a quantidade de ovos de $M$. domestica predada varia com o tipo de substrato e o tempo decorrido após a última tomada de alimento.

Segundo KINN (1966) a temperatura de $25^{\circ} \mathrm{C}$ favo rece a ação predatória e aumenta a fecundidade de $M$. muscaedomesticae em 1elação às temperaturas de $30^{\circ}$ e $20^{\circ} \mathrm{C}$.

AXTELL (1961) reportou que, por dia, 2,7 ovos

FILIPPONI, A. Sulla natura dell'associazione trà Macrocheles muscaedomesticae et Musca domestica. Riv. Parassitol., 16: 83-102, 1955. 
e larvas $\left(L_{1}\right)$ de moscas foram destruídas por fêmea de $M$. mus caedomesticae, quando oferecidas sobre papel úmido. Utilizan do recipientes $(120 \times 120 \times 20 \mathrm{~cm})$ contendo fezes de gado leiteiro, o autor obteve uma redução de $94 \%$ na população de moscas no recipiente com 20.000 ovos de mosca, 200 fêmeas de $M$. muscaedomesticae e 200 fêmeas de Glyptholaspis confusa (Foa), (Macrochelidae) en relação à testemunha, contendo somente 20.000 ovos de moscas (AXTELL, 1963a). O'DONNELL \& AXTELL (1965) adicionaram 100 ovos de mosca a $20 \mathrm{M}$. muscaedomestical adultos em um reci piente $(210 \mathrm{~m} \ell)$ contendo o meio SMA e água. A mortalidade ob tida foi de $96,1 \pm 1,52 \%$ após 72 horas.

SINGH et alii (1966) obtiveram 84,5\% de contro le de M. domestica e 91,9\% de F. canicularis, com un äcaro pa ra cinco moscas, em aproximadamente $16 \mathrm{~cm}^{3}$ de excremento boví no e $150 \mathrm{mg}$ de ave, respectivamente. Os autores relataram que M. muscaedomesticae exposto simultaneamente a igual número de ovos de moscas diferentes, destruiu 2,3 ovos de M. domestica, 1,4 de $F$. canicularis e 1,0 de $M$. autummalis em 60 minutos.

AXTELL (1963a) observou que ácaros da família Uropodidae freqüentemente ocorriam em excremento de gado lei teiro e de aves, no Estado de New York, sugerindo uma runção predatōria para estes ācaros.

O'DONNELL \& AXTELL (1965) determinaram a taxa predatória para Fuscoropoda vegetans (Uropodidae). Sobre papel de filtro umedecido 9,5 ovos e larvas $L_{l}$ de mosca domés- 
tica foram destruídos por machos de $F$. vegetans; 11,6 por fêmeas e 9,4 por deutoninfas, em 7 dias.

O'DONNELL \& NELSON (1967) compararam a ação prẹ datória de $F$. vegetans e M. muscaedomesticae em ovos de $F$. ca nicularis, enquanto que WILLIS \& AXTELL (1968) determinaram o potencial destes dois ācaros como agentes controladores de $M$. domestica

G. confusa (Foa) foi relatado pela primeira vez na América do Norte por AXTELL (1961). O autor comparou a atividade predatória de fêmeas deste ácaro e outros três ácaros Macrochelidae, com ovos de mosca doméstica expostos em papel de filtro umedecido. G. confusa destruiu em média 9,9 ovos e larvas $I_{1}$ por dia, Macrocheles rubustalus (Berlese)4,6; M. muscaedomesticae 2,7 e Macrocheles subbadius (Berlese) 1,2. Em excremento de gado leiteiro G. confusa apresentou-se como - quarto ácaro Macrochelidae mais abundante, seguido de M. mus caedomesticae, Macrocheles merdarius (Berlese) e M. subbadius (AXTELL, 1963b).

Os resultados obtidos dos estudos realizados por PECK (1969) quanto ao potencial de preaação foram relativamente insignificantes para Fuscuropoda sp. e G. confusa e bastante significativos para M. muscaedcuesticae.

PECK \& ANDERSON (1969) observaram os seguintes ācaros predadores em esterco de aves: G. confusa, M. glaber, M. muscaedomesticae e Fuscuropoda sp.. 


\subsection{MÉTODOS DE COLETA}

Na extração de inúmeros artrópodes das amostras de excremento animal, vários autores têm se utilizado do funil de Tullgren (PECK \& ANDERSON, 1969; 1970; PFEIFFER \& AXTELL, 1980; LEGNER et alii, 1981).

Funil de Berlese-Tullgren ou Funil de Tullgren é uma combinação do funil de cobre aquecido, elaborado pelo entomologista italiano A. Berlese e subseqüentemente modifica do pelo sueco A. Tullgren, que utilizou uma lâmpada como fonte de calor e de luz, direcionando os indivíduos para um tubo coletor contendo álcool (LEWIS \& TAYLOR, 1967; SOUTHWOOD, 1968).

MOORE (1954) utilizou o método de flotação na obtenção de artrópodes adultos. o autor comparou seu método com a técnica de extração do Berlese e considerou-o mais eficiente.

LEGNER \& OLTON (1970) observaram a fauna de es terco animal de várias regiões do mundo utilizando o método de flotação. o método foi designado para coletar somente os individuos adultos.

GEETHA BAI \& SANKARAN (1977) tambēm coletaram adultos dos parasitóides, predadores e outros artrópodes atra vés de flotação. Os imaturos foram coletados manualmente. 


\section{MATERIAL E MÉTODOS}

\subsection{DESCRIGÃO DA GRANJA}

o levantamento foi conduzido na Granja Mizumoto, localizada na região de Bastos, Estado de são Paulo, situada à distância de $6 \mathrm{~km}$ da zona urbana. Ocupa uma área de 53 alq, contendo 182.000 aves distribuidas em galinhei ros. Cada galinheiro, estruturado totalmente em madeira, abri ga de 1.200 a 3.000 aves. Estas permanecem na granja por um período de até dois anos, após os quais são comercializadas e suas instalações são mantidas vazias por um período de um mês, durante o qual são limpas, desinfetadas e todo excremento do so 10, eliminado. Para maior controle das atividades, as instalações são reuniảas em seções, constituídas de 10 a 12 galinheiros.

As fezes frescas, acumuladas sob as gaiolas, constituem a fonte mais importante de criação de moscas nos aviārios. A produção é da ordem de 1.000 a $2.700 \mathrm{~kg}$ mensais por seção, sendo comum sua comercialização cora aảubo orgânico. Praticamente nenhuma medida é tomada, referente ao controle de mosca, pois situada numa região avícola, qualquer esforço è neutralizado pela constante reinfestação. 


\subsection{AMOSTRAGEM}

Amostras semanais, do excremento acumulado sob as gaiolas, foram tomadas no decorrer de dezesseis (16) meses, no período de setembro de 1985 a dezembro de 1986 .

A escolha do local amostrado foi determinada de maneira aleatória, através de sorteios semanais. A cada sema na, seis (6) locais foram amostrados. O acompanhamento da tem peratura foi realizado diariamente com um termômetro de máxima e minima.

Encerraram-se as amostragens quando as aves fo ram removidas das instalações.

As amostras foram tomadas com um cilindro de po lietileno $(10,0 \mathrm{~cm}$ de diâmetro por $22,0 \mathrm{~cm}$ de altura) que foi inserido no esterco e retirado com o auxílio de uma espátula. Ocasionalmente, para fins comerciais, a empresa retirava - excremento, impossibilitando a amostragem.

o material coletado foi armazenado em recipien tes plásticos bem vedados e enviado ao laboratório para a extração da fauna.

\subsection{EXTRAGÃo DA FAUNA dO EXCREMENTO AMOSTRADO}

A separação da fauna, do excremento, era realí zada em funil de Tullgren modificado, onde a amostra permane- 
cia por um período de cinco (5) dias. Durante este periodo, ocasionalmente, o material era umedecido, favorecendo o proces so de extração. A amostra ao ser descartada, no quinto dia, era examinada e eventualmente algum material era coletado, evi tando perdas.

o funil de Tullgren modificadoé constituído de um funil de plástico (200 cm de diâmetro superior); uma penei ra $(0,5 \mathrm{~cm}$ de malha), onde o material era depositado; um recí piente coletor contendo álcool $70 \%(0,5$ litro) e uma lâmpada de 40 watts sobre o material a uma distância de $\pm 12,0 \mathrm{~cm}$. A bateria, formada por seis (6) unidades, como descrita acima, era sustentada por uma armação de madeira e coberta com papel aluminio.

\subsection{TRIAGEM E IDENTIFICAGÃO}

Apōs a extração do material, realizou-se uma triagem preliminar com peneiras do tipo "Granutest" e, posteriormente, com lupa estereoscópica. Todos os componentes da fauna, exceto ácaros, eram numerados.

A identificação do material foi efetuada por comparação com exemplares do Museu de Zoologia de São Paulo, através de chaves de identificação e pelos especialistas: Dr. Howard Frank, Universidade da Flórida, Gainesville, EUA- Coleoptera, Staphylinidae; Dr. Rupert L. Wenzel, "Field Mu- 
seum of Natural History", Chicago, EUA - Coleoptera, Histeridae; Dr. Carlos H.W. Flechtmann, Departamento de Zoologia, ESALQ/USP, Piracicaba-SP - Acarina.

Os espēcimes identificados foram depositados no Museu do Departamento de Entomologia da ESALQ/USP. 


\title{
4. RESULTADOS E DISCUSSÃO
}

\author{
4.1. DIPTERA
}

\subsubsection{Adulto}

Durante os dezesseis meses de amostragem foram coletados 9.662 dipteros adultos, considerando apenas a subordem Brachycera. Uma vez que o método de coleta não é adequa do para coletar adultos de dípteros, a alta incidência pode ser explicada pela atração exercída pelo funil de Berlese (luz e odor), pela captura direta de adultos no excremento e pelos adultos emergidos de pupas das amostras.

Muscidae foi a familia de maior ocorrência, re presentada pelas espécies: Musca domestica, Stomoxys calcitrans, Muscina stabulans e Fannia canicularis, totalizando 52,97\% dos dípteros coletados (Tabela 1). Entre as espécies de dipteros, M. domestica foi a mais abundante no levantamento $(52,15 \%)$ 'Tabela2). Este alto nümero de espécimens pode ser resultante das condições ideais para o desenvolvimento deste inse to nos locais de coleta. 
Tabela 1. Familias de dípteros adultos coletados em excremento de aves. Número de indivíduos coletados e porcentagem. Bastos-SP, setembro/1985 a dezembro/1986.

Família

No indivíduos
Porcentagem

(응
Muscidae

Sphaeroceriäae

Chloropidae

Sarcophagiāae

Drosophylidae

Stratiomyidae

Calliphoridae

Sepsidae

Scenopinidae

Phoridae

Syrphidae
5.118

3.923

259

143

115

66

13

10

8

6

1
52,97

40,60

2,68

1,48

1,19

0,68

0,13

0,10

0,08

0,06

0,01

TOTAL

9.662

100 
o número de moscas adultas de $S$. calcitrans co letadas foi muito baixo. Segundo GUIMARÃES (1984) estas moscas utilizam o excremento de aves como substrato para o desenvolvimento larval, porém os adultos deslocam-se para āreas de criação de gado, do qual se alimentam.

M. stabulans foi encontrada em menor nümero, talvez devido ao fato de se desenvolver em fezes de consistência semi-líquida. Segundo GREENBERG \& POVOLNY larvas do primeiro ínstar parecem ser mais saprófagas em rela ção aos demais instares, que são predadores particularmente a larva de terceiro Instar de larvas de outros dípteros. Entretanto, segundo GUIMARÃES (1988), esta atividade é limitada e parece não ser de importância no controḷe biológico de $M$. domestica e outras espécies.

A segunda maior familia, com 3.923 espécimes $(40,60 \%)$ foi Sphaeroceridae (Tabela 1). Leptocera ferruginata apresentou-se como a espécie mais numerosa da família e a segunda entre todos os dípteros coletados, constituindo 35,25\% (Tabela 2). Com menor expressão, os demais dipteros coletados representaram outras nove (9) familias (Tabela 1). 
Tabela 2. Espēcies mais abundantes de dípteros adultos coleta dos em excremento de aves. Bastos-SP, setembro/1985 a dezembro/1986.

\begin{tabular}{llcc}
\hline Espécie & Família & Individuos & $\begin{array}{c}\text { Porcentagem } \\
(\%)\end{array}$ \\
\hline Musca domestica & Muscidae & 5.039 & 52,15 \\
Leptocera ferruginata & Sphaeroceridae & 3.406 & 35,25 \\
Leptocera sp. & Sphaeroceridae & 449 & 4,65 \\
Hippelates sp. & Chloropidae & 259 & 2,68 \\
Wohlfartia sp. & Sarcophagidae & 136 & 1,41 \\
\hline
\end{tabular}

Ovos quebrados e aves mortas constituem rica fonte alimentícia, bastante atrativa a inúmeros insetos. Ade mais, a presença de gramíneas entre os galinheiros, resulta em um excelente refúgio justificando, assim, a ocorrência de muitos insetos que não obrigatoriamente se desenvolvem em excremento de aves.

A maioria das espécies de Drosophylidae, por exemplo, criam-se em frutos em decomposição e em fungos, embo ra sejam encontrados próximas de estrumes. Larvas de muitas espécies de Chloropidae vivem em hastes de gramíneas, enquan to que outras espécies vivem em matéria decomposta.

Segundo SANDERS \& DOBSON (1966), adultos de Sphaeroceridae e Sepsidae são bastante freqüentes ao redor de montes de esterco. As larvas vivem em excremento animal e em vărias matérias em decomposição. Este substrato é tambēm 
atrativo para as espēcies das famílias Phoridae e Syrphidae.

A presença de Calliphoridae e Sarcophagidae em aviārios è principalmente decorrente da existência de aves mortas. As larvas são geralmente necrófagas, vivendo em carne putrefata, excremento e matérias semelhantes. PECK \& ANDERSON (1970) registraram que em excremento, com uma semana de idade, Calliphoridae são tambëm muito abundantes.

A ocorrència de Scenopinidae (Scenopinidus sp.) em excremento é devido a existência de fungos e de larvas de outros insetos. Segundo KELSEY (1981) e PETERSON (1960), lar vas de Scenopinidae são predadoras e se alimentam de uma variedade de insetos.

Freqüentemente encontrado em aviários, o estra tiomídeo Hermetia illucens apresentou uma população adulta bastante numerosa durante todo o levantamento, diminuindo sen sivelmente nos períodos mais frios. Não obstante, um pequeno número de adultos foi coletado. Este fato está relacionado com o porte avantajado do inseto, que dificulta na passagem pela peneira, com o longo período de desenvolvimento pupal e com o mētodo de coleta.

\subsubsection{Imaturo}

A família Muscidae, perfazendo $66,42 \%$ das larvas amostradas, foi a mais abundante, seguida pela família Stratiomyidae com 25,76\% (Tabela 3). 
Tabela 3. Famílias e espécies de dípteros, estágio larval, co letados em excremento de aves. Número de indivíduos coletados e porcentagem. Bastos-SP, setembro/ 1985 a dezembro/1986.

\begin{tabular}{llcc}
\hline Familia & Espécie & $\begin{array}{c}\text { No de } \\
\text { individuos }\end{array}$ & $\begin{array}{c}\text { Porcenta- } \\
\text { gem (\%) }\end{array}$ \\
\hline Musciāae & $\begin{array}{l}\text { Musca domestica } \\
\text { Stomoxys calcitrans }\end{array}$ & 57.010 & \\
& Fannia canicularis & 91.045 & 66,42 \\
Stratiomyidae & Hermetia illucens & 22.870 & 25,76 \\
Sphaeroceriōae & Leptocera spp. & 2.437 & 2,74 \\
Calliphoridae & Phormia regina & 1.867 & 2,10 \\
Syrphidae & & 1.071 & 1,20 \\
Sepsidae & Sepsis spp. & 783 & 0,88 \\
\end{tabular}

M. domestica $(64,78 \%)$ e H. illucens $(25,76 \%)$ foram as espécies mais numerosas. Observando a porcentagem men sal destas larvas, è possivel detectar que à medida que a população de H. illucens aumentou, a população de M. domestica diminuiu (Tabela 4). 
Tabela 4. Purcentagem de larvas de M. domestica e H. illucens, coletadas em excremento de aves. Bastos-SP, setembro 1985 a dezembro 1986.

\begin{tabular}{|c|c|c|c|c|c|c|c|c|c|c|c|c|c|c|c|c|}
\hline Diptero periodo & Set. & out. & Nov. & Dez. & Jan. & Fer. & Mar. & Abr. & $\mathrm{Mai}$. & תמנת. & Jul. & Ago. & Set. & out. & Nov. & Dez. \\
\hline $\begin{array}{l}\text { lusca } \\
\text { domestica }\end{array}$ & 79,83 & 26,20 & 8,89 & 9,76 & 12,03 & 13,82 & 39,34 & 84,44 & 25,99 & 6.28 & 30,70 & 40,02 & 32,91 & 59,15 & 61,08 & $25, \bar{\varepsilon}$ \\
\hline $\begin{array}{l}\text { Hermetia } \\
\text { iliucens }\end{array}$ & 1,25 & 4,04 & 54,54 & 51,32 & 59,01 & 40,79 & 11,99 & 0,89 & 5,77 & 0,99 & 9,48 & 0,62 & 0,05 & 0,65 & 0,07 & $0, \Xi \dot{0}$ \\
\hline $\begin{array}{l}\text { Temperatura } \\
\text { mëäia }\left({ }^{\circ} \mathrm{C}\right)\end{array}$ & 26,0 & 27,6 & 28,8 & 29,1 & 28,8 & 27,2 & 27,5 & 26,9 & 24,8 & 22,7 & 21,3 & 22,6 & 24,3 & 26,3 & 28,9 & $27, \varepsilon$ \\
\hline
\end{tabular}

OBS.: Foi testada a regressão polinomial entre temperatura e número de insetos (Musca domestica e Hermetia illucens) e não foi encontrada significância atē 4 ? grau. 
Segundo GREENBERG \& POVOLNY (1971) e De BACH (1964) la $\underline{\text { }}$ vas de H. illucens podem sob certas condições, exercer controle natural significativo sobre a reprodução de $M$. domestica, não por predação, mas por competição. Compete com a mosca em seu desenvolvimento larval, tornando o meio liquefeito, que è ina dequado para o desenvolvimento de $M$. domestica. Assim sendo, quando a população de $H$. illucens aumenta, a população de $M$. domestica diminui. FURMAN et alii (1959) sugerem competição por alimento ou espaço como sendo o mecanismo de interação en tre Musca e Hermetia e segundo BRADLEY \& SHEPPARD (1984) hā algum tipo de comunicação química interespecífica.

\subsection{COLEOPTERA}

Mais de quinze (15) familias de coleópteros fọ ram encontradas em excremento de aves, em Bastos-SP. Algumas famílias coletadas são conhecidas como predadoras e outras co mo escavadoras. Hā famílias, entretanto, que estão relaciona das com cereais secos, fungos ou são simplesmente de ocorrência acidental.

As familias mais abundantes de coleópteros adul tos coletadas foram: Histeridae, Curculionidae, Tenebrionidae, Staphylinidae, Cucujidae e Mycetophagidae (Tabela 5). Contudo, representantes das seguintes famílias tambēm foram amostrados: Trogidae, Nitidulidae, Anthicidae, Anobiidae, Scolytidae, Elä teridae, Hydrophilidae, Dermestidae, Scarabaeidae, Carabidae e Rhizophagidae. 
Segundo WALLACE \& TYNDALE-BISCOE (1983), a ação do complexo faunístico de esterco (Scarabaeidae, Hydrophilidae, Histeridae e ácaros) provoca alta taxa de mortalidade em imaturos de Musca vetustissima.

Na fase larval, Tenebrionidae foi a família mais numerosa (5.718 larvas) e Histeridae a segunda (3.012 larvas). Juntas representaram 91,43\% das 9.547 larvas amostradas. Foram coletadas tambēm larvas das famílias Dermestidae, Trogidae e Staphylinidae.

Tabela 5. Familitias mais abundantes de coleóptros adultos coletados em excremento de aves. Nümero de indivíduos e porcentagem. Bastos-SP, setembro/1985 a dezembro/1986.

\begin{tabular}{lcc}
\hline Familia & No individuos & $\begin{array}{c}\text { Porcentagem } \\
(\%)\end{array}$ \\
\hline Histeridae & 2.093 & 36,12 \\
Curculionidae & 745 & 12,86 \\
Tenebrionidae & 741 & 12,79 \\
Staphylinidae & 626 & 10,81 \\
Cucujidae & 485 & 8,37 \\
Mycetophagidae & 459 & 7,92 \\
\hline
\end{tabular}

A familia Hiscieridae (Tabela 5), a mais numero sa e com ocorrència durante todo levantamento, foi representa da por seis (6) espécies: Carcinops troglodytes (Payk.); Eus pilotus arrogans (Mars.); Euspilotus modestian, Acritus analis Lec.; Leionata reichei; Hister sp. 
PFEIFFER \& AXTELL (1980) E PECK \& ANDERSON (1969) coletaram em excremento de aves outras espécies de histerideos que não estes.

Segundo o Dr. Rupert Wenzel (comunicação pessoal) a espécie A. analis é pela primeira vez relatada para o Brasil.
C. troglodytes foi a espécie mais abundante, representando $14,62 \%$ dos 5.793 coleópteros coletados (Tabela 6).

Tabela 6. Espécies mais abundantes de coleópteros, coletados em excremento de aves. Número de indivíduos e porcentagem. Bastos-SP, setembro/1985 a dezembro/ 1986.

\begin{tabular}{lllc}
\hline Espécies & Familia & No individuos & $\begin{array}{c}\text { Porcentagem } \\
(\%)\end{array}$ \\
\hline $\begin{array}{lll}\text { Carcinops } \\
\text { troglodytes (Payk.) }\end{array}$ & Histeridae & 847 & 14,62 \\
Sitophilus oryzae & Curculionidae & 748 & 12,91 \\
Alphitobius diaperinus & Tenebrionidae & 730 & 12,60 \\
Euspilotus arrogans & Histeridae & 564 & 9,74 \\
Typheae sp. & Mycetophagidae & 459 & 7,92 \\
Cryptolestes pusillus & Cucujidae & 273 & 4,71 \\
Euspilotus modestian & Histeridae & 235 & 4,06 \\
Acritus analis & Histeridae & 233 & 4,02 \\
Hister sp. & Histeridae & 205 & 3,54 \\
Laemophoeus testaceus & Cucujidae & 200 & 3,45 \\
\hline
\end{tabular}


A alta freqüência do curculinídeo Sitophilus oryzae pode ser atribuída à utilização do milho no alimento das aves.

A presença de A. diaperinus foi bastante acentuada, principalmente no estágio larval. Segundo RAMIREz \& VACCA (1981) A. diaperinus influi significativamente na diminuição do peso e umidade do esterco, devido seu hábito de cavar túneis, aumentando a circulação do ar, tornando o habitat desfavorâvel para o desenvolvimento das larvas e moscas.

Os estafilinídeos constituíram 10,81\% dos coleópteros e foram representados por doze (12) espécies: Aleochara puberula Klug.; Philanthus sordidus (Grav.); Philanthus sp.; Neohypnus ettenuatus (Erichson); Neohypnus sp.; oxytelus sculptus Grav.; Tinatus sp.A; Tinatus sp.B; Acrotoma sp.; Lathrobium sp.; Apheloglossa sp. e Scopaeus sp..

LEGNER \& OLTON (1970) registraram a ocorrência de A. puberula e $P$. sordidus, porém em excremento bovino.

A presença de cucujideos neste meio decorre do uso de ração, a qual freqüentemente atacam, kein como cereais e grãos armazenados.

A familia Mycetophagidae, foi representada por uma espécie, Typhaea sp. Segundo Arnett (1963) ${ }^{7}$, citado por

7 ARNETT, R.H. The beetles of the United States. The Catholic University of America Press, Washington, D.C., 1963. 1112p. 
SHUBECK et alii (1981), é provável que estes coleópteros alimentem-se exclusivamente de fungos.

\subsection{OUTRAS ORDENS}

No decorrer da pesquisa, duas espēcies não iden tificadas de Dermaptera foram coletadas, porēm numa quantidade muito pequena em relação a que era observada no excremen to. o método de coleta aplicado não foi adequado para capturar estes insetos, que mostram grande agilidade quando perturbados.

LEGNER \& OLTON (1970) coletaram quatro espēcies de Dermaptera em excremento bovino e LEGNER et alii (1981) duas espécies. Em excremento de aves LEGNER (1971) obteve um Labiduridae, Eubarellia annulipes.

Espécies de Anthocoridae (Hemiptera) também fo ram coletadas. Segundo LEGNER \& OLTON (1970), adultos de ento mófagos e saprófagos da fauna de excremento animal pertencem principalmente às ordens Coleoptera, Dermaptera e Hemiptera, as quais estão comumentes associadas aos locais de oviposição e alimentação larval de dípteros.

Espécimens da familia Formicidae foram coletadas, porēm não identificados. Em excremento bovino, GEE THA BAI \& SANKARAN (1977) observaram duas espécies de Formicí 
dae (Solenopsis gemminata e Dorylus orientalis) predando : Iarvas de mosca.

\subsection{OUTROS ARTRÓPODES}

As familias de ácaros coletadas foram: Macrochelidae, Uropodidae, Acaridae e Cheyletidae. Entretanto, não foi possivel a identificação a nível de espécie.

Macrochelidae compreende um grupo de predadores cuja distribuição e capacidade predatória tem sido bastan te discutida (WADE \& RODRIGUEZ, 1961 e AXTELL, 1963a,b e 1968).

Foram coletadas espécimens de pseudoescorpião (Pseudoscorpionida). GEETHA BAI \& SANKARAN (1977) e LEGNER et alii (1981) registraram tambēm a presença de pseudoescorpião em excremento animal. 


\section{CONCLUSÕES}

Com base nos resultados obtidos, pode-se con cluir que:

- Existe um complexo de artrōpodes predadores de Musca doméstica em excremento de aves.

- o uso de cilíndro para fazer a amostragem não è adequado para a coleta de todos os tipos de artröpodes presentes no excremento.

- Musca ciomestica (Muscidae) sofre competição de Hermetia illucens (Stratiomyidae) em excremento de aves. 


\section{REFERÊNCIAS BIBLIOGRÁFICAS}

ABBOTT, C.E. The necrophilous habit in coleoptera. Bulletin of the Brooklyn Entomological Society, Brooklyn, 32: 2024,1937 .

ANDERSON, J.R. \& POORBAUGH, J.H. Observation on the ethology and ecology of various diptera associated with Northern California poultry ranches. Journal of Medical Entomology, Honolulu, $\underline{1}(2): 131-47,1964 \mathrm{a}$.

ANDERSON, J.R. \& POORBAUGH, J.H. Biological control possibị lity for house flies. California Agriculture, Berkeley, 18 (9) : 2-4, $1964 \mathrm{~b}$.

AXTELL, R.C. New records of North American Macrochelidae(Aca rina:Mesostigmata) and their predation rates on the house fly. Annals of the Entomological Society of America, Columbus, $\underline{54}: 748,1961$.

AXTELL, R.C. Effect of Macrochelidae (Acarina:Mesostigmata) on house fly production dairy cattle manure. Journal of Economic Entomology, Menasha, 56: 317-21, 1963a.

AXTELI, R.C. Acarina occurring in domestic animal manure. Annals of the Entomological Society of American, Columbus, $\underline{56}(5): 628-33,1963 \mathrm{~b}$. 
AXTELL, R.C. Integrated house fly control, population of fly larvae and predaceous mites, Macrocheles muscaedomesticae, in poultry manure after larvicide treatment. Journal of Economic Entomology, Menasha, 61(1): 245-9, 1968.

BALDUF, W.V. The bionomics of entomophagous coleoptera. St. Louis, John S. Swift, 1935. V.1.

BRADLEY, S.W. \& SHEPPARD, D.C. House fly oviposition inhibition by larvae of Hermetia illucens, the black soldier fly. Journal of Chemical Ecology, Georgia, 10(6): 853-9, 1984 .

DAVIS, W.T. Silpha surinamensis and creophilus villosus as predaceous insects. Journal of the New York Entomological Society, New York, 23: 150-1, 1915.

DEBACH, P. Biological control of insect pests and weeds. New York, Reinhold, 1964. 844 p.

DILLON, E.S. \& DILLON, L.S. A manual of common beetles of Eastern North America. New York, Dover, 1972. 2v.

EVANS, M.E.G. A comparative account of feeding methods of the beetles Nebria brevicollis (F.) (Carabidae) and Philonthus decorus (Grav.) (Staphylinidae). Transactions of the Royal Society of Edinburg, Edinburg, 66: 91-109, 1964.

FICHTER, G.S. Necrophily vs. necrophagy . Ohio Journal Science, Columbus, 49 : 201-4, 1949 . 
FURMAN, D.P.; YOUNG, R.D.; CATTS, E.P. Hermetia illucens (Linnaeus) as a factor in the natural control of Musca domestica Linnaeus. Journal of Economic Entomology, Menasha, 르(5): 917-21, 1959 .

GEETHA BAI, M. \& SANKARAN, T. Parasites, predators and other arthropods associated with Musca domestica and other flies breeding in bovine manure. Entomophaga, Paris, 22(2): 1637,1977 .

GREENBERG, B. \& POVOLNY, O. Bionomics of flies. In: Flies and disease. Ecology, classification and biotic associations. New Jersey, Princenton, 1971. v.1, p. 56-83.

GUIMARÃES, J.H. Mosca dos estábulos, uma importante praga do gado. Agroquímica Ciba-Geigy, São Paulo, 23: 10-4, 1984 .

GUIMARÃES, J.H. Perspectivas de controle integrado de mosca sinantrópica em aviários no Estado de São Paulo. In: GUIMARÄES, J.H. coord., Seminārio sobre controle de Artrópodes de importância veterinária, Campinas, Fundação Cargill, 1988. p.1-8

KELSEY, L.P. Scenopinidae. In: MCALPINE, J.F.; PETERSON, B. V.; SHEWELL, G.E.; TESKEY, H.J. VOCKEROTH, J.R.; WOOD, D. M. coord., Manual of nearctic Diptera, Ottawa, Biosystematics Research Institute, 1981. v.1, p. 525-8.

KINN, D.N. Predation by the mite, Macrocheles muscaedomest $i-$ cae (Acarina:Macrochelidae), on three species of flies. Journal of Medical Entomology, Honolulu, 3: 155-8, 1966. 
LEGNER, E.F. Some effects of the ambient arthropod complex on the density and potential parasitization of muscoid diptera in poultry wastes. Journal of Economic Entomology, Menasha, 64 (1): 111-5, 1971 .

LEGNER, E.F. \& BRYDOM, H.W. Suppression of dung-inhabiting fly populations by pupal parasites. Annals of the Entomological Society of America, Columbus, 59(4): 638-51, 1966 .

LEGNER, E.F. \& OLTON, G.S. Worldwide survey and comparison of adult predator and scavenger insect populations associated with domestic animal manure where livestock is artificially congregated. Hilgardia, Berkeley, $\underline{40}(9): 225-66,1970$.

LEGNER, E.F.; GREATHEAD, D.J.; MOORE, I. Equatorial East African predatory and scavenger arthropods in bovine excre ment. Environmental Entomology, College Park, 10: 620-5, 1981 .

LEGNER, E.F.; OLTON, G.S.; EASTWOOD, R.E.; DIETRICK, E. J. Seasonal density distribution and interactions of predatory and scavenger arthropods in accumulating poultry wastes in coastal and interior Southern California. Entomophaga, Pa ris, $20(3): 269-83,1975$.

LEWIS, T. \& TAYLOR, L.R. Experimental ecology. London, Academic Press, 1967. 401p.

MANK, H.G. The biology of the staphylinidae. Annals of the Entomological Society of America, Columbus, 16:220-37, 1923.

MOORE, I. An efficient method of collecting dung beetles. Pan Pacific Entomologist, San Francisco, 30: 208, 1954. 
O'DONNELL, A.E. \& AXTELL, R.C. Predation by Fuscoropoda vegetans (Acarina:Uropididae) on the house fly (Musca domestica). Annals of the Entomological Society of America, Columbus, 58: 403-4, 1965.

O'DONNELL, A.E. \& NELSON, E. Predation by Fuscoropoda vegetans (Acarina:Uropodidae) and Macrocheles muscaedomesticae (Acarina:Macrochelidae) on the eggs of the little house fly, Fannia canicularis. Journal of the Kansas Entomological Society, Manhattan, 40: 441-3, 1967.

PECK, J.H. The potential role of arthropod predators in the integrated control of Diptera developing in poultry droppings. Berkeley, 1968. I29p. (MS. University of California)

PECK, J.H. Arthropod predators of immature diptera developing in poultry droppings in Northern California. Part II. Laboratory studies of feeding baheviours and predation potential of selected species. Journal of Medical Entomology Honolulu, $6(2): 168-71,1969$.

PECK, J.H. \& ANDERSON, J.R. Arthropod predators of immature diptera developing in pultry droppings in Northern California. Part I. Determination, seasonal abundance and natural cohabitation with prey. Journal of Medical Ento-

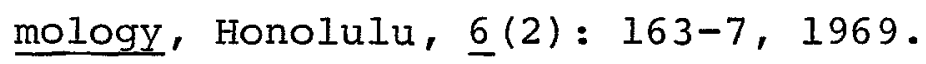

PECK, J.H. \& ANDERSON, J.R. Influence of poultry manure removal schedules on various diptera, larvae and selected arthrofud predators. Journal of Economic Entomology, Menasha, 63: (1) : 82-90, 1970 . 
PEREIRA, C. \& DE CASTRO, M.P. Contribuição para o conhecimento da espēcie tipo de Macrocheles Lotr. (Acarina): $M$. muscaedomesticae (Scopoli, 1772).emend. Arquivo do Instituto Biolćgico de São Paulo, São Paulo, 16: 153-86, 1945.

PETERSON, A. Larvae of insects: an introduction to neartic species. Columbus, 1960. v.2, p.250-1.

PFEIFFER, D.G. \& AXTELL, R.C. Coleoptera of poultrymanure in caged-layer houses in North California. Environemntal Entomology, College Park, 9: 21-8, 1980.

PIMENTEL, D. Relationship of ants to fly control in puerto Rico. Journal of Economic Entomology, Menasha, 48(1): 2830,1955 .

RAMIREZ, B.F. \& VACCA, T.R. Influencia del cucarrón Alphitobius diaperinus Panzer (Coleoptera-Tehebrionidae) em el secamiento del estierco de gallinas enjauladas. Tolima, 1981. 43p. (Tesis - Medicina Veterināria (Ibague) Univer sidad del Tolima - Colombia).

RODRIGUEZ, J.G. \& WADE, D.F. The nutrition of Macrocheles muscaedomesticae (Acarina: Macrochelidae) in relation to its predatory action on the house fly egg. Annals of the Entomological Society of America, Columbus, 54: $782-8$, 1961 .

SANDERS, D.P. \& DOBSON, R.C. The insect complex associated with bovine manure in Indiana. Annals of the Entomological Society of America, Columbus, 59(5): 955-9, 1966. 
SHUBECK, P.P.; DOWNIE, N.M.; WENZEL, R.L.; PECK, S.B. Species composition and seasonal abundance of carrion beetles in an Oak-Beech forest in the great swamp national wildlife refuge (N.J.). Entomological News, Philadelphia, g(l): 716,1981 .

SIMMONDS, H.W. Investigations with a view to the biological control of house flies in Fiji. Tropical Agriculture, London, 17: 197-9, 1940.

SINGH, P.; KING, E.W.; RODRIGUEZ, J.G. Biological control of muscids as influenced by host preference of Macrocheles muscaedomesticae (Acarina: Macrochelidae). Journal of Medical Entomology, Honolulu, 3: 78-81, 1966 .

SOUTHWOOD, T.R.E. Ecological methods. London, Methuen, 1968. $391 \mathrm{p}$.

XAMBEAU, P. Moeurs et metamorphoses. Philonthus. Le Naturaliste, Paris, 29: 115-7, 144-6, 1907.

WADE, C.F. \& RODRIGUEZ, J.G. Life history of Macrocheles muscaedomesticae (Acarina: Macrochelidae) in relation to predatory action on the house fly egg. Annals of the Entomological Society of America, Columbus, $\underline{54}(6): 776-81$, 1961 .

WALIACE, M.M.H. \& TYNDALE-BISCOE, M. Attempts to measure the influence of dung heetle (Coleoptera-Scarabaeidae) on the field mortality of the bush fly Musca vetustissima Walker (Diptera-Muscidae) in Sutheastern Australia. Bulletin of Entomological Research, Wallingford, $73(1): 33-4,1983$.

WILLIS, R.R. \& AXTELI, R.C. Mite predators on the house fly, a comparison of Fuscuropoda vegetans and Macrocheles muscaedomesticae. Journal of Economic Entomology, Menasha, $\underline{61}(6): 1669-74,1968$. 